\title{
Glycyrrhetinic acid and its derivatives in infectious diseases
}

\author{
Dominik Langer ${ }^{1}$, Beata Czarczynska-Goslinska ${ }^{2}$, Tomasz Goslinski ${ }^{1 *}$ \\ ${ }^{1}$ Department of Chemical Technology of Drugs, Poznan University of Medical Sciences, Grunwaldzka 6, 60-780 Poznan, Poland \\ ${ }^{2}$ Department of Pharmaceutical Technology, Poznan University of Medical Sciences, Grunwaldzka 6, 60-780 Poznan, Poland
}

\begin{tabular}{l}
\hline ARTICLE INFO \\
\hline Received 21 March 2016 \\
Accepted 22 April 2016
\end{tabular}

Keywords:

glycyrrhetinic acid,

licorice,

infectious diseases.

\begin{abstract}
Introduction. Licorice or liquorice (Glycyrrhiza glabra, Leguminosae) is a perennial plant naturally occurring or cultivated in Europe and Asia. It was appreciated by many ancient cultures, and was employed within Arabic medicine and (beginning in the Middle Ages) in Europe folk medicine as a remedy for many diseases. Currently, the sweet flavoured root of this plant - Radix Glycyrrhizae (Liquirtiae), is widely taken for the treating of various upper respiratory tract diseases, as well as for gastric ulcer disease. It is also utilized as a sweetening and flavouring agent in the food, tobacco and pharmacy industries. The main active ingredient of liquorice is the triterpenoid saponin, glycyrrhizin, which is a mixture of calcium, magnesium and potassium salts of glycyrrhizic acid (GA). Glycyrrhizic acid is composed of an aglycone, that is $18 \beta$-glycyrrhetinic acid (GE), and a D-glucuronic acid dimer. The aim of this review is to discuss some aspects of the activity of glycyrrhetinic acid and its derivatives in infectious diseases.

State of knowledge. The pentacyclic system of glycyrrhetinic acid consists of condensed six-membered rings with a hydroxyl group at C-3, a carboxyl moiety at C-30 and a ketone functional group at $\mathrm{C}-11$. Considering the presence of the above mentioned functional groups, many structural transformations have been proposed, including those by way of esterification, alkylation and reduction reactions. The introduction of various chemical residues into its structure, as well as the modification of the glycyrrhetinic acid in its pentacyclic triterpene skeleton, has led to the generation of compounds with many valuable antimicrobial, anti-parasitic, antiviral properties and modified lipophilic parameters.

Summary. In summary, glycyrrhetinic acid derivatives appear to have promise as active pharmaceutical ingredients that contain a wide range of biological and pharmacological properties.
\end{abstract}

\section{INTRODUCTION}

Licorice or liquorice (Glycyrrhiza glabra, Legumino$s a e)$ is a perennial plant naturally occurring or cultivated in Europe (the Mediterranean) and Asia (especially the central and South-Western parts). It was used in ancient Assyrian, Egyptian, Chinese and Indian cultures and appreciated by ancient Greeks and Romans. In addition, it was used in Arabic medicine, and, from the Middle Ages onwards, in Germany, Italy, Spain and England as a remedy for ailments in the respiratory, gastrointestinal, cardiovascular, genital-urinary systems, as well as for skin, eye and other diseases $[9,10]$.

Currently, the root of this plant - Radix Glycyrrhizae (Liquirtiae), which has a sweet flavor, is widely used for

\footnotetext{
* Corresponding author

e-mail: tomasz.goslinski@ump.edu.pl
}

treating upper respiratory tract diseases and gastric ulcer disease. It is also employed as a sweetening and flavouring agent in the food, pharmacy and tobacco industries, respectively $[16,7]$.

\section{MEDICINALCHEMISTRYANDPHARMACOLOGICAL ACTIVITY OF GLYCYRRHETINIC ACID AND ITS ANALOGUES}

The main active ingredient of liquorice is the triterpenoid saponin, glycyrrhizin, which is a mixture of calcium, magnesium and potassium salts of glycyrrhizic acid. It is present in concentrations that are mainly of $1-9 \%$ (up to $15 \%$ ), depending on the species, geographical location and methods of extraction. Glycyrrhizic acid (GA) 1 is composed of $18 \beta$-glycyrrhetinic acid (GE) 2 , an aglycone that is 
a pentacyclic triterpene derivative of the $\beta$-amyrin type, and D-glucuronic dimer $\mathbf{3}$, which is linked to the aglycone via a glycosidic bond (Fig. 1) [8,41].

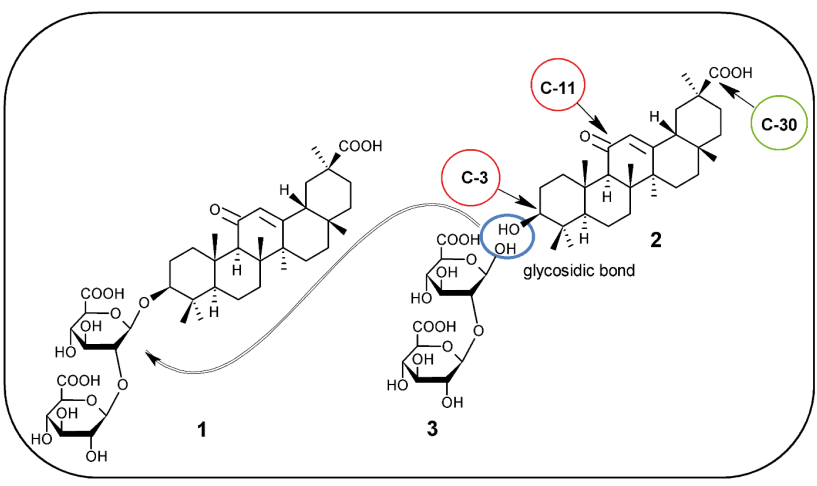

Figure 1. Glycyrrhizic acid (GA) 1 is composed of an aglycone that is glycyrrhetinic acid (GE) 2, and glucuronic acid dimer 3, which is linked to the aglycone via a glycosidic bond. Herein, a glycosidic bond is formed between the hemiacetal group of $\mathbf{3}$ and the hydroxyl group of aglycone $\mathbf{2}$

The pentacyclic system of GE molecule 2 consists of condensed six-membered rings with a hydroxyl group at C-3, a carboxyl moiety at C-30 and a ketone functional group at $\mathrm{C}-11$. In addition, $\mathrm{GE}$ exists in the form of two $18 \alpha$ (trans) and $18 \beta$ (cis) stereoisomers which have different stability and solubility in acidic, alkaline solutions or organic solvents (especially dioxane, isopropanol or ethylene glycol) $[40,44]$. The chemical structure of the GE molecule gives great possibilities for modifications leading to derivatives. This was reviewed by Tolstikov et al. [40] and Kang et al. [21]. Considering the presence of the above mentioned functional groups at C-3, C-11 and C-30, many structural transformations have been proposed for generating new drugs, including esterification, alkylation and reduction reactions $[14,21,40]$.

The bioactivity and potential health benefits of licorice have been lately reviewed by Kao et al. [22]. GA or GE, and in some cases, both acids, have exerted in many in vitro and in vivo studies, antiulcer [2], anti-inflammatory [2], hepatoprotective [17], anticancer [37,47], antibacterial [25], antiviral $[3,10,13]$, antileishmanial $[6,42]$ and neuroprotective activities, as well as cell-protective and chemopreventive effects $[1,50]$. Moreover, GE and its analogues have demonstrated anticoagulant activity as inhibitors of blood coagulation factor $\mathrm{Xa}$, and have shown antifilarial properties against microfilariae and adult worms of Brugia malayi $[18,19]$. GE and its analogues are also known, due to their antiobesity activity, as inhibitors of adipogenic differentiation via the phosphorylation of protein kinase B/Akt, and as stimulators of lipolysis through hormone-sensitive lipase/ HSL activation [29]. Today, products are being marketed that are based on the favorable pharmacological properties of the active ingredients of Glycyrrhiza glabra L. These include e.g. formulations of glycyrrhizin for patients with liver disease (Stronger Neo-Minophagen C, SNMC) [10], and the GE anti-inflammatory formulation, Phytosome ${ }^{\circledR}$ $[15,36]$.

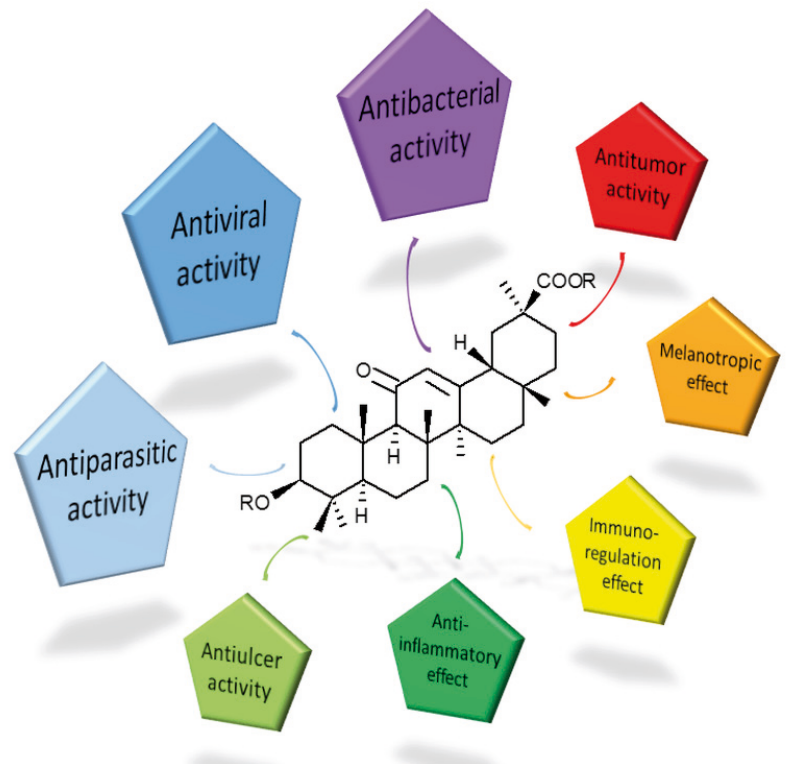

Figure 2. Types of pharmacological activity of glycyrrhetinic acid and glycyrrhizic acid

\section{ANTIVIRAL ACTIVITY OF GLYCYRRHETINIC ACID AND ITS ANALOGUES}

It is not surprising that the age-old need for treating virus-induced diseases has brought about a search for antiviral drugs within the plant world [5]. Hence, the antiviral properties of licorice root extracts have been explored and exploited by mankind since ancient times [4,10,48]. Among other studies, the antiviral effects of Glycyrrhiza glabra L. species were reviewed by Fiore et al. [10]. In this work, glycyrrhizin and its derivatives revealed in vivo or in vitro activity against hepatitis $\mathrm{B}$ and $\mathrm{C}$ viruses, herpes simplex virus, influenza $A$, human immunodeficiency virus type 1 (HIV-1), severe acute respiratory syndrome related coronavirus, respiratory syncytial virus, vesicular stomatitis virus, vaccinia virus and arboviruses. Moreover, according to Harada et al. [12], it was, as well, possible to bring about an interference with HIV-1 reverse transcriptase by way of the use of GE at molecular biology levels.

It is also noteworthy that oleanolic acid, asiatic acid, and the derivatives of glycyrrhetinic and oleanolic acids have shown activity against EV71. Human enterovirus 71 (EV71) was identified as the most frequent causative agent of hand, foot, and mouth disease (HFMD). The effects of glycyrrhetinic acid derivatives modification at the $\mathrm{C}-3$ position with D-phenylglycine were examined by way of application to EV71 virus-infected human rhabdomyosarcoma cells 4 (Fig. 3). Herein, the inhibitory effects of this derivative on EV71 replication was analysed by reverse transcription (RT)-PCR and Western blot analysis. The results of such efforts showed that rhabdomyosarcoma cells incubated with a GE derivative demonstrated ca. 35\% decrease in viral transcripts. Furthermore, Western blot studies also revealed decreased levels of viral proteins e.g. for viral protein VP1. In this work, ca. $80 \%$ reductions were observed [49]. 


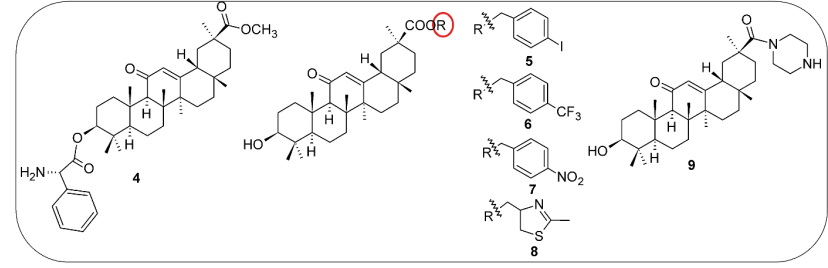

Figure 3. Structures of glycyrrhetinic acid derivatives 4-9

Further synthetic development of GE analogues have led to $\mathrm{C} 3$ substituted spacer-bridged derivatives. These compounds have revealed interesting antiviral activity in vitro against the influenza A virus infection. Herein, analogues of the triterpene saponin glycyrrhizin containing 1-thio- $\beta$ D-glucuronic acid residues were assessed via biological study on a cell line infected with influenza A virus. What is more, the cytotoxicity of compounds was assessed on Madin-Darby Canine Kidney Epithelial Cells (MDCK) treated with compounds in concentrations from $3.1 \mu \mathrm{M}$ to $250 \mu \mathrm{M}$. It is interesting that, in this study, none of the compounds tested was toxic at concentrations up to 250 $\mu \mathrm{M}$, whereas glycyrrhetinic acid and the hemisuccinate carbenoxolone showed $\mathrm{CC}_{50}$ values of $7.4 \mu \mathrm{M}$ and $17.8 \mu \mathrm{M}$, respectively. The antiviral activity of the GE derivatives was determined by infecting MDCK cells with influenza A H3N2 virus and simultaneously treating them with different concentrations of GE derivatives. In such work, the 3-(2-thioethyl)-N-acetylamino- and 3-(2-thioethyl)-thiolinked glucuronide GE derivatives prevented virus-induced cytopathicity and restored cell viability, when compared to virus-infected control cells. Both GE derivatives were found to be effective inhibitors. The $\mathrm{IC}_{50}$ values for the 3-(2-thioethyl)-N-acetylamino- and 3-(2-thioethyl)-thiolinked glucuronide GE derivatives were found to be $87 \mu \mathrm{M}$ and $54 \mu \mathrm{M}$, respectively [39].

GE analogues have exhibited high activity against several DNA viruses. Kumada et al. [26] and Van Rossum et al. [45] reported the potential of GE against Hepatitis C virus (HCV), while Wang et al. [46] presented the synthesis, biological evaluation and structure-activity relationships of GE derivatives against Hepatitis B virus (HBV). In such studies, GE derivatives activity against HBV were assessed by measurement of the stage of the viral DNA replication cycle in HepG 2.2.15 cells, and applications of GE analogues were compared with $\mathrm{GE}$ as a reference $\left(\mathrm{IC}_{50}=39.28\right.$ $\mu \mathrm{M})$ and the nucleoside drug, tenofovir, as a positive control $\left(\mathrm{IC}_{50}=0.98 \mu \mathrm{M}\right)$. The results of such work (via biological testing) have revealed that sixteen compounds exhibited higher inhibitory activities against HBV DNA replication than did GE with $\mathrm{IC}_{50}$ values less than $10 \mu \mathrm{M}$. Among these, the GE analogues (5-8) (Fig. 3) showed significant inhibitions of HBV DNA at $\mathrm{IC}_{50}=5.71,5.36,8.90,9.08 \mu \mathrm{M}$, respectively. Furthermore, cytotoxicity tests of GE and its analogues showed that high toxicity of GE can be lowered by esterification of the hydroxyl group at C-3, or by way of the carboxyl group at C-30. However, the modification of both functional groups may lead to the reduction of antiviral potential. The introduction of a suitable substituent to the carboxyl group located at C-30 allowed the drug to increase the activity against HBV DNA replication of 5-8. However, the introduction of the epoxide functionality at the C-12 (13) double bond resulted in a decrease in the antiviral properties of GE derivatives, as two effects were observed. In this case, the inhibiting effect of HBV DNA replication with $\mathrm{IC}_{50}$ to $18.37 \mu \mathrm{M}$ was accompanied by an increase in cytotoxicity, with $\mathrm{CC}_{50}=35.71 \mu \mathrm{M}$. Unfortunately, molecular mechanisms of 5-8 antiviral activities in cells was not completely evaluated [46].

\section{ANTIBACTERIAL ACTIVITY OF GLYCYRRHETINIC ACID AND ITS ANALOGUES}

In recent years, the antimicrobial activity of plant extracts and phytochemicals has been evaluated against both antibiotic susceptible and resistant microorganisms. Furthermore, the synergistic effect for association of natural products isolated from plants with antibiotics was ascertained. Plants have been, for ages, a valuable source of natural products with known antimicrobial properties. Therefore, the use of plant extracts and phytochemicals possessing known antimicrobial properties can be of significance in therapy [30]. In work of this nature, glycyrrhetinic acid was recognized as a compound possessing antimicrobial properties $[24,28,32,35]$.

Licorice root extracts tested using the agar diffusion method have shown significant antibacterial activities against gram-positive (Bacillus subtilis, Staphylococcus aureus) and gram-negative bacteria (Escherichia coli, Pseudomonas aeruginosa) [31]. This observation corresponds to another study, where GE demonstrated antibacterial activity against Bacillus subtilis and Staphylococcus epidermidis at 7.6 and $12.5 \mu \mathrm{g} /$ $\mathrm{ml}$, without inducing hemolysis of human erythrocytes. However, at that time, no inhibitory activity of GE was found against E. coli, Proteus vulgaris, and against various fungi such as Candida albicans, Saccharomyces cerevisiae or Trichosporon beigelii. Still, GE was seen to inhibited the synthesis of DNA, RNA and proteins without membrane disruption [24]. Noteworthy is that liquorice and glycyrrhizin have been used for ages in the treatment of peptic ulcers. Herein, the potent in vitro activity of GE against various Helicobacter pylori isolates has provided a further explanation for its positive effect on peptic ulcers. In such research, the majority of the total of $29 \mathrm{H}$. pylori strains (ca. 79.3\%) were inhibited at a low concentration of $\leq 50 \mathrm{mg} / \mathrm{L}$. Especially interesting and promising was the revelation that GE showed effectiveness against clarithromycin-resistant strains. This can be considered as the basis for an alternative therapeutic agent against these pathogens [25]. In addition, in other work, the antibacterial effects of GE were studied on periodontopathogenic and capnophilic bacteria. This revealed good efficacy [35].

It is noteworthy that GE has been found to inhibit the survival of methicillin-resistant S. aureus (MRSA), and that it attenuates virulence gene expression. In such studies, it was put forward that the bactericidal activity of GE against MRSA may be related to the inhibition of expression of selected virulence genes, among these, saeR and hla [28]. In a further study, evaluation was performed of the glycyrrhetinic acid activity against 55 hospital strains of $S$. aureus and 32 hospital strains of Actinobacillus 
actinomycetemcomitans isolated from patient specimens by culture methods. In this work, GE demonstrated high bacteriostatic and bactericidal potential, and Minimum Inhibitory Concentration (MIC) values for $S$. aureus and A. actinomycetemcomitans were found at 64 and $8 \mu \mathrm{g} / \mathrm{ml}$, respectively, whereas Minimum Bactericidal Concentration (MBC) were found at 64 and $16 \mu \mathrm{g} / \mathrm{ml}$ [34].

Recent studies have shown that GE and its piperazine analogue modified at the C-30 position of GE (9) (Fig. 3), in combination with isoniazid, rifampicin and streptomycin, was effective as an anti-tuberculosis agent. In addition, the synergistic effect of $18 \beta-\mathrm{GE}$ or compound 9 with these drugs has led to the significantly decreasing of the MIC of isoniazid, rifampicin, streptomycin and $18 \beta-\mathrm{GE}$ or its derivative, against drug-resistant Mycobacterium bovis. Still, the anti-tuberculosis mechanism of GE was not found. The above data are encouraging enough to consider GE derivatives as potential anti-bacterial compounds [51].

\section{ANTIPARASITIC ACTIVIY OF GLYCYRRHETINIC ACID AND ITS ANALOGUES}

Many natural plant-derived products including alkaloids, terpenes and phenolics, have revealed anti-parasitic properties of surprising efficacy and selectivity. This antiparasitic potential has been seen in the laboratory and represent lead structures for the development of modern and urgently needed anti-parasitics. The terepenes, in particular, have shown activity against certain parasites e.g. simple monoterpenes (espintanol), iridoids (amarogentin), sesquiterpenes (artemisinin), diterpenes (cryptotanshinone) and limonoids (nimbolide) [23]. With regard to GE, its antiparasitic activity has been made evident, and its effectiveness in anti-malarial, anti-leishmanial and other treatments has been reported in the experimental studies described below.

Malaria is an infectious and life-threatening disease caused by the protozoan parasites belonging to the genus Plasmodium. While the anti-malarial mechanism of GE is yet unknown, molecular modeling studies have indicated that GE may be a competitive inhibitor of Plasmodium falciparum lactate dehydrogenase enzyme. This effect is similar to that of chloroquine, a standard antimalarial drug. GE was identified as a lead candidate for further detailed in-silico, biological and pharmaceutical investigations. In these studies, the effect of GE on the innate immune system in malaria infected mice is being ascertained, while combination studies of GE with established anti-malarials are being carried out against drug resistant strains to increase or revoke the efficacy of the resistant anti-malarial drugs [20].

The major tool to combat leishmaniasis infection is chemotherapy. There are, unfortunately, no vaccines available. What is more, some of the drugs employed in the primary treatment of leishmaniasis (such as pentavalent antimonials) often pose problems due to toxicity to liver and heart tissues and because of other severe adverse effects. Yet, through on-going investigation, many plant-derived natural products have been recognized as potential bioactive agents with anti-protozoal and anti-inflammatory activities. Therefore, there is constant interest in generating safe and specifically targeted drugs against infectious diseases [33]. In such studies, GE was found to trigger curative Th1 response and nitric oxide up-regulation in an experimental visceral leishmaniasis that is associated with the activation of protein complex NF- $\kappa \mathrm{B}$. In one study, an 11-fold increase in NO production was observed, with $20 \mu \mathrm{M} \mathrm{GE}$ in mouse peritoneal macrophages infected with Leishmania donovani promastigotes. Herein, it was suggested that the therapeutic effect of GE may be associated with modulation of the kinase/phosphatase balance or with the induction of pro-inflammatory mediators production through the activation of a protein complex NF- $\kappa \mathrm{B}$ in parasite infected cells. It was also noted in such work that GE induced NF- $\kappa$ B migration into the nucleus of parasite-infected cells, and it brought about a diminished presence of $\mathrm{I} \kappa \mathrm{B}$ in the cytoplasm. Furthermore, it was found that GE exerts an effect at some level upstream of I $\mathrm{B}$ kinase (IKK) in the signaling pathway, and that it induces the production of proinflammatory mediators through a mechanism related to the induction of NF- $\kappa \mathrm{B}$ activation. The marked sensitivity of Th macrophage effector responses of NO and proinflammatory cytokines to GE suggests that this natural product could be used as a potential immunomodulator in order to generate the required immunity [42]. In another study, it was shown that the kinase/phosphatase balance plays an important role in modulating infection. Therefore, the effect of GE on MAPK (mitogen-activated protein kinase) directed phosphatases (MPK) targeted for additional research. In this, it was seen that GE markedly reduced the expression and activities of three phosphatases, MKP1, MKP3 and protein phosphatase 2A (PP2A), along with engendering a substantial reduction of spleen parasite burden, and being associated with a concomitant decrease of individual phosphatase levels [43]. The latest research has indicated the existence of a GE induced mediated effect upon kinase p38 (which belongs to the MAPK family), mitogen-activated protein kinase kinase 3 (MKK3) and MKK6. These being immediate upstream regulators of $\mathrm{p} 38$. Further studies have identified the transforming growth factor $\beta$-activated kinase 1 (TAK1), myeloid differentiation factor 88 (MyD88), interleukin 1 receptor (IL-1R)-activated kinase 1 (IRAK1), and tumor necrosis factor (TNF) receptor-associated factor 6 (TRAF6) to be important contributors to GE-mediated macrophage activation. Finally, the activity and the mechanism of action of glycyrrhetinic acid against leishmaniasis was ascertained to have connections with the Toll-like receptor 2 (TLR2) and TLR4. In summary, the antileishmanial activity of glycyrrhetinic acid was found to be dependent on the TLR2/4-MyD88 signaling axis, leading to MKK3/6-mediated canonical and TAB1-mediated noncanonical p38-NF- $\kappa$ B activation [11].

Recent studies have shown that due to the structural modification of glycyrrhetinic acid, interesting GE analogues of a favourable profile of action against microfilarial may be obtained. This is of importance because it gives the potential to treat lymphatic filariasis, which is a longstanding chronic disease caused by Wuchereria bancrofti, Brugia malayi and Brugia timori, transmitted through the bites of infected mosquitoes. Currently anti-filarial therapies including diethylcarbamazine, ivermectin and albendazole are less effective towards adult parasites. Therefore, GE derivatives 
offer many opportunities for finding anti-parasite drugs. In such work, the introduction of the acyl group in place of the hydroxyl group at C-3 has been found to reduced the potential against microfilariae, in comparison to GE $(10,11)$ (Fig. 4). Moreover, the esterification of the carboxyl group at the C-30 has been revealed to slightly increase antifilarial activity $(12,13)$, as compared to 10 and 11 . However, the substitution of the carboxyl group with the $\mathrm{C}-30$ amide group alone has led to compounds of satisfactory parameters $(14,15)$. Herein, both 14 and 15 showed adulticidal activity in vivo against $B$. malayi in birds (mariones unguiculatus) [19].

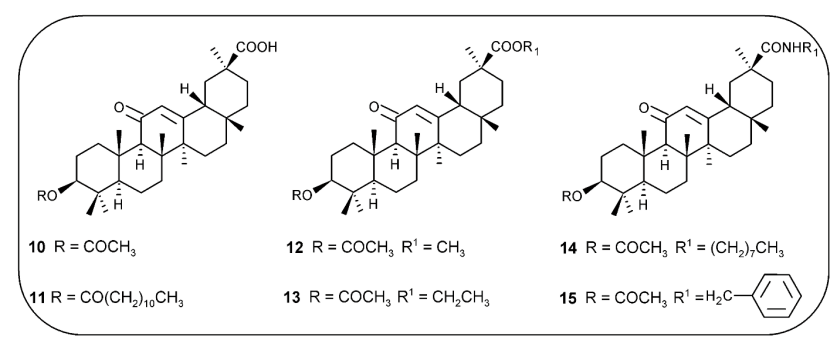

Figure 4. Structures of glycyrrhetinic acid derivatives 10-15

\section{CONCLUSIONS}

Plant saponins possess a wide range of biological and pharmacological properties. Some of these are well established [27,38,40,44], and the structure active relationships of GE and its synthetic derivatives have been the topic of on-going study around the world. The introduction of various chemical residues, as well as the modifications made to the GE pentacyclic triterpene skeleton, have led to the creation of compounds demonstrating many valuable antimicrobial, antiparasitic and antiviral properties. These activities have also led to generating compounds with modified lipophilic parameters. To sum up, GE derivatives appear to be promising candidates for the creation of drugs inclusive of a wide range of biological and pharmacological properties.

\section{ACKNOWLEDGEMENT}

This research was financed from a statutory grant from the Poznan University of Medical Sciences.

\section{REFERENCES}

1. Akman T. et al.: The Neuroprotective Effect of Glycyrrhizic Acid on an Experimental Model of Focal Cerebral Ischemia in Rats. Inflammation, 38, 1581, 2015.

2. Aly A.M., Al-Alousi L., Salem H.A.: Licorice: A Possible Antiinflammatory and Anti-ulcer Drug AAPS PharmSciTech., 6, 74, 2005.

3. Ashfaq U.A. et al: Glycyrrhizin as antiviral agent against Hepatitis C Virus. J. Transl. Med., 9, 1, 2011.

4. Asl M.N., Hosseinzadeh H.: Review of Pharmacological Effects of Glycyrrhiza sp. and its Bioactive Compounds. Phytother. Res., 22, 709, 2008.

5. Baltina L.A. et al.: Search for new drugs prospects for the creation of new antiviral drugs based on glycyrrhizic acid and its derivatives (a review). Pharm. Chem. J., 43, 539, 2009.

6. Bhattacharjee S. et al.: Glycyrrhizic acid suppresses Cox-2-mediated anti-inflammatory responses during Leishmania donovani infection. J. Antimicrob. Chemother., 67, 1905, 2012.
7. Carmines E.L., Lemus R., Gaworski C.L.: Toxicologic evaluation of licorice extract as a cigarette ingredient. Food Chem. Toxicol., 43, 1303, 2005.

8. Fenwick G.R., Lutomski J., Nieman C.: Liquorice, Glycyrrhiza glabra L. Composition, Uses and Analysis. Food Chem., 38, 119, 1990.

9. Fiore C. et al.: A history of the therapeutic use of liquorice in Europe, J. Ethnopharmacol., 99, 317, 2005.

10. Fiore C. et al.: Antiviral Effects of Glycyrrhiza species. Phytother. Res., 22, 141, 2008.

11. Gupta P., Das P.K., Ukil A.: Antileishmanial Effect of $18 \beta$-Glycyrrhetinic Acid Is Mediated by Toll-Like ReceptorDependent Canonical and Noncanonical p38 Activation Antimicrob. Agents Chemother., 59, 2531, 2015.

12. Harada S. et al.: Biochemical characterisation of recombinant HIV-1 reverse transcriptase (rRT) as a glycyrrhizin-binding protein and the CK-II-mediated stimulation of rRT activity potently inhibited by glycyrrhetenic acid derivate. Biol. Pharm. Bull., 21, 1282, 1998.

13. Hardy M.E. et al.: $18 \beta$-glycyrrhetinic acid inhibits rotavirus replication in culture. Virol. J., 9, 1, 2012.

14. Hu J., Wu J., Ju Y.: A dual-responsive macrocycle based on glycyrrhetinic acid. Tetrahedron Lett., 53, 6705, 2012.

15. Indena. Industria Derivati Naturali. www.indena.com/ pdf/18beta_18betaPhyto_st_pc.pdf (access: 2016.01.02).

16. Isbrucker R.A., Burdock G.A.: Risk and safety assessment on the consumption of Licorice root (Glycyrrhiza sp.), its extract and powder as a food ingredient, with emphasis on the pharmacology and toxicology of glycyrrhizin. Regul. Toxicol. Pharmacol., 46, 167, 2006.

17. Jeong H.G. et al.: Hepatoprotective effects of $18 \beta$-glycyrrhetinic acid on carbontetrachloride-induced liver injury: inhibition of cytochrome p450 2e1 expression. Pharmacol. Res., 46, 221, 2002.

18. Jiang L. et al.: Discovery of glycyrrhetinic acid as an orally active, direct inhibitor of blood coagulation factor xa. Thromb. Res., 133, 501, 2014.

19. Kalani K. et al.: Glycyrrhetinic acid and its analogs: A new class of antifilarial agents. Bioorg. Med. Chem. Lett., 23, 2566, 2013.

20. Kalani K. et al.: In Silico and In Vivo Anti-Malarial Studies of $18 \beta$ Glycyrrhetinic Acid from Glycyrrhiza glabra. PLoS One, 8, 1, 2013.

21. Kang L. et al.: Research Progress on Structure Modification and Biological Activity of $18 \beta$-Glycyrrhetinic Acid, Curr. Opin. Complement. Alternat. Med., 1, 34, 2014.

22. Kao T.C., Wu C.H., Yen G.C.: Bioactivity and Potential Health Benefits of Licorice. J. Agric. Food Chem., 62, 543, 2014.

23. Kayser O., Kiderlen A.F., Croft S.L.: Natural products as antiparasitic drugs, Parasitol. Res., 90, S55, 2003.

24. Kim H.K. et al.: Antimicrobial mechanism of $\beta$-glycyrrhetinic acid isolated from licorice, Glycyrrhiza glabra Biotechnol. Lett., 24, 1899, 2002.

25. Krausse R. et al.: In vitro anti-Helicobacter pylori activity of Extractum liquiritiae, glycyrrhizin and its metabolites, J. Antimicrob. Chemother., 54, 243, 2004.

26. Kumada H.: Long-term treatment of chronic hepatitis $\mathrm{C}$ with glycyrrhizin [Stronger Neo-Minophagen C (SNMC)] for preventing liver cirrhosis and hepatocellular carcinoma, Oncology, 62, 94, 2002.

27. Lacaille-Dubois M.A., Wagner H.: A review of the biological and pharmacological activities of saponins, Phytomedicine, 2, 363, 1996.

28. Long D.R. et al.: 18 $\beta$-Glycyrrhetinic Acid Inhibits MethicillinResistant Staphylococcus aureus Survival and Attenuates Virulence Gene Expression, Antimicrob. Agents Chemother., 57, 241, 2013.

29. Moon M.H. et al.: $18 \beta$-Glycyrrhetinic acid inhibits adipogenic differentiation and stimulates lipolysis, Biochem. Biophys. Res. Commun., 420, 805, 2012.

30. Nascimento G.G.F. et al.: Antibacterial activity of plant extracts and phytochemicals on antibiotic-resistant bacteria. Braz. J. Microbiol., 31, 247, 2000.

31. Nitalikar M.M. et al.: Studies of antibacterial activities of glycyrrhiza glabra root extract. Int. J. Pharm.Tech. Res., 2, 899, 2010.

32. Pellati D. et al.: In vitro Effects of Glycyrrhetinic Acid on the Growth of Clinical Isolates of Candida albicans. Phytother. Res., 23, 572, 2009.

33. Rodrigues I.A. et al.: Natural Products: Insights into Leishmaniasis. Inflammatory Response. Mediators Inflamm., 2015, 1, 2015. 
34. Salari M.H., Eshraghi S., Noroozi M.: Antibacterial effects of glycyrrhetinic acid on 55 hospital strains of Staphylococcus aureus and 32 actinobacillus actinomycetemcomitans. DARU, 9, 37, 2001.

35. Salari M.H., Kadkhoda Z.: In vitro antibacterial effects of glycyrrhetinic acid on periodontopathogenic and capnophilic bacteria isolated from adult periodontitis. Clin. Microbiol. Infect., 9, 987, 2003.

36. Semalty A. et al.: Supramolecular phospholipids-polyphenolics interactions: The $\mathrm{PHYTOSOME}^{\circledR}$ strategy to improve the bioavailability of phytochemicals. Fitoterapia, 81, 306, 2010.

37. Smolarczyk R. et al.: The Role of Glycyrrhizin, an Inhibitor of HMGB1 Protein, in Anticancer Therapy. Arch. Immunol. Ther. Exp., 60, 391, 2012.

38. Sparg S.G., Light M.E., van Staden J.: Biological activities and distribution of plant saponins. J. Ethnopharmacol., 94, 219, 2004.

39. Stanetty C. et al.: Synthesis and antiviral activities of spacer-linked 1-thioglucuronide analogues of glycyrrhizin. Beilstein J. Org. Chem., $8,705,2012$.

40. Tolstikov A., Baltina A., Serdyuk N.G.: Search for new drugs: Glycyrrhetic acid (a review). Pharm. Chem. J., 32, 5, 1998.

41. Tykarska E., Czarczyńska-Goslińska B., Lulek J.: Kwas glicyryzynowy i glicyretynowy w nowoczesnej technologii farmaceutycznej. Czas. Aptek., 19, 43, 2012.

42. Ukil A. et al.: Leishmaniasis Associated with the Activation Up-Regulation in Experimental Visceral Th1 Response and Nitric Oxide 18ß-Glycyrrhetinic Acid Triggers Curative. J. Immunol., 175, $1161,2005$.

43. Ukil A. et al.: Curative Effect of 18b-Glycyrrhetinic Acid in Experimental Visceral Leishmaniasis Depends on PhosphataseDependent Modulation of Cellular MAP Kinases. PLoS One, 6, 1 , 2011.
44. Vampa G., Benvenuti S.: Separtion of $18 \alpha$ - and $18 \beta$-glycyrrhetinic acid by high-performance thin-layer chromatographic densitometry. J. Chromatogr. A, 543, 479, 1991.

45. Van Rossum T.G. et al.: Glycyrrhizin induced reduction of ALT in Europea n patients with chronic hepatitis C. Am. J. Gastroenterol., 96, 2432, 2001.

46. Wang L.J. et al.: Synthesis, biological evaluation and structureactivity relationships of glycyrrhetinic acid derivatives as novel antihepatitis B virus agents. Bioorg. Med. Chem. Lett., 22, 3473, 2012.

47. Yamaguchi H. et al.: Novel effects of glycyrrhetinic acid on the central nervous system tumorigenic progenitor cells: Induction of actin disruption and tumor cell-selective toxicity. Eur. J. Med. Chem., 45, 2943, 2010

48. Yeh C.F. et al.: Water extract of licorice had anti-viral activity against human respiratory syncytial virus in human respiratory tract cell lines. J. Ethnopharmacol., 148, 466, 2013.

49. Zhao C.H. et al.: Inhibition of human enterovirus 71 replication by pentacyclic triterpenes and their novel synthetic derivatives. Chem. Pharm. Bull., 62, 764, 2014.

50. Zhou J. et al. $18 \beta$-glycyrrhetinic acid suppresses experimental autoimmune encephalomyelitis through inhibition of microglia activation and promotion of remyelination. Sci. Rep., 5, 1, 2015.

51. Zhou X. et al.: Antimycobacterial and Synergistic Effects of $18 \beta$ Glycyrrhetinic Acid or Glycyrrhetinic acid-30-piperazine in Combination with Isoniazid, Rifampicin or Streptomycin against Mycobacterium bovis. Phytother. Res., 26, 253, 2012. 